\title{
A TRIO OF MODULATORS FOR THE FERMILAB TEVATRON ELECTRON LENS PROJECT
}

\author{
D. Wildman, V. Shiltsev, N. Solyak, FNAL*, Batavia, IL 60510 \\ V. Efanov, P. Yarin, FID Technology, St. Petersburg, Russia
}

\section{Abstract}

Three high voltage modulators used during testing and operation of the Tevatron Electron Lens (TEL) at Fermilab will be described. Short high voltage ( 0 to $\sim 20 \mathrm{kV}$ ) pulses from these modulators vary the anode-cathode voltage of the TEL electron gun to control the magnitude of the electron beam current. The trio of modulators include a low repetition rate MOSFET-based pulser, a fast ionization device, and a high average power tetrode modulator The characteristics of each device will be discussed and typical outputs from each type of modulator will be shown.

\section{INTRODUCTION}

In the Tevatron Collider Run II at Fermilab, 36 bunches of protons and antiprotons collide head on at the two interaction regions B0 and D0 and suffer longer range collisions at other points in the ring as the two beams spiral around each other in helical orbits. Differences in proton beam intensities and the spacing of the beams into three groups of 12 bunches will create a beam-beam tune shift that is different for each of the 36 antiproton bunches. The goal of the Tevatron Electron Lens (TEL) Project is to compensate these tune shifts of the antiproton bunches on an individual bunch by bunch basis. This is done by passing an electron beam of varying intensity through the antiproton beam over a $2 \mathrm{~m}$ long interaction region.

The TEL Project recently installed the first of two electron lenses in the Tevatron [1]. This first lens has already demonstrated the ability to shift the tune of a single proton bunch circulating in the accelerator. The magnitude of the tune shift is proportional to the current density in the electron beam. To adjust the electron current in the beam, the voltage difference between the electron gun [2] anode and cathode electrodes is modulated. The lens vacuum beam pipe and diagnostic pick-ups are all grounded. The gun cathode potential is held at some negative potential, typically $-10 \mathrm{kV}$ to $-13 \mathrm{kV}$. Ideally, we would like to be able to modulate the electron current from zero to its maximum value of $3-5 \mathrm{~A}$ during the $395 \mathrm{~ns}$ spacing between antiproton bunches. However; simulations [3] indicate that this level of modulation will not be necessary, since the difference in tune shifts between adjacent antiproton bunches is only a fraction of the total range of tune shifts required.

*Operated by Universities Research, Inc. under contract with the U.S. Department of Energy

\section{MOSFET SWITCH}

The first modulator tested was a PVX-4110 10kV pulse generator manufactured by Directed Energy, Inc. This device is actually two HV switches consisting of parallel and series combinations of power MOSFETs. The HV output is selected between the two HV inputs using a TTL logic level control gate. The two inputs may be any voltage between $-10 \mathrm{kV}$ and $+10 \mathrm{kV}$. The pulse width is adjustable from $<140 \mathrm{~ns}$ to dc by setting the length of the control gate. The pulse rise and fall times are less than 40ns. The peak output current cannot exceed 15A and the maximum average output power, which is defined as the energy switched per cycle times the repetition rate, cannot exceed $100 \mathrm{~W}$. Although the pulser can operate at $50 \mathrm{kHz}$ with bursts up to several $\mathrm{MHz}$, this average power limitation will not allow the pulser to be operated at the full $10 \mathrm{kV}$ output at a $50 \mathrm{kHz}$ rate. Figure 1 shows the output pulse from this modulator switching between ground and $3.5 \mathrm{kV}$.

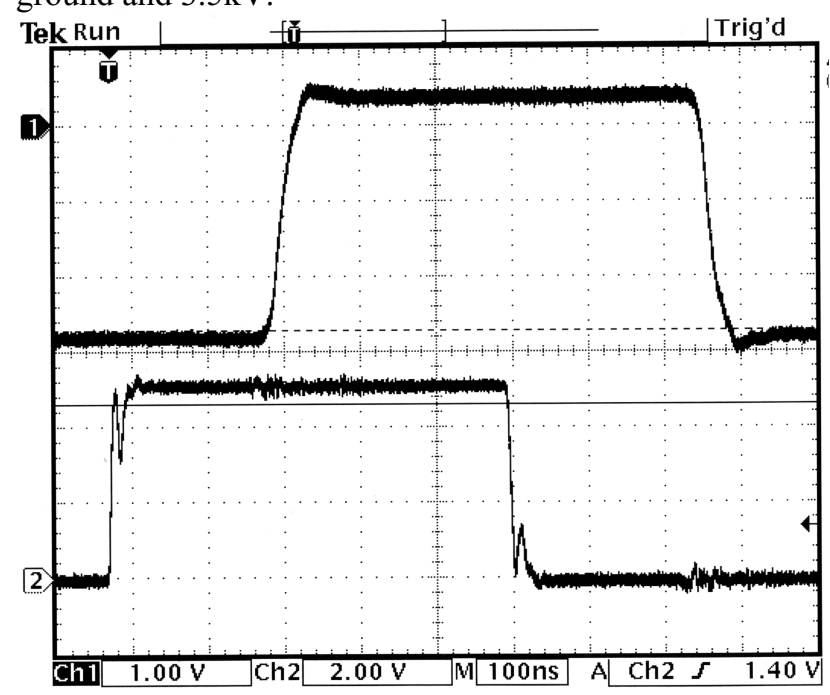

Figure 1: MOSFET switch control gate input signal (lower trace) and output pulse $(1 \mathrm{kV} /$ div $)$ at a time scale of $100 \mathrm{~ns} / \mathrm{div}$.

This pulser or switch is ideal for charging capacitive loads, such as the electron gun anode, since the load capacitance is directly connected to the high voltage power supply without any charging resistor. This switch is especially well suited for bench tests of the electron gun. The gun anode can be biased at the negative cathode potential (HV Input 1 ) then switched to another negative voltage near ground (HV Input 2 ) to initiate the electron beam current pulse. 


\section{FAST IONIZATION PULSER}

The second modulator tested was $20 \mathrm{kV}, 50 \mathrm{~ns}$ wide pulser (model \# FPG20-50S) designed and manufactured by FID Technology. The pulser uses a combination of fast $(<1 \mathrm{~ns})$ closing Fast Ionization Device (FID) switches and fast opening Drift Recovery Diodes (DRD) to generate the output pulses [4]. The pulser is arranged into four identical blocks whose outputs are then combined. Each block has a single DRD shunting its output connector. The DRD is connected by coaxial cables to two LC circuits, each LC circuit having its own stack of FID switches. The pulsing sequence begins when one of the two FID stacks closes, exciting the first LC circuit and sending current through the conducting DRD. After a half cycle oscillation in the first LC circuit, the current reversed direction and the second FID stack closes, exciting the second LC circuit. During this time charge is being pumped out of the DRD. When all of the charge has been pumped out, the DRD opens, interrupting the large current flow and generating a $5 \mathrm{kV}, 50 \mathrm{~ns}$ pulse at the output. .

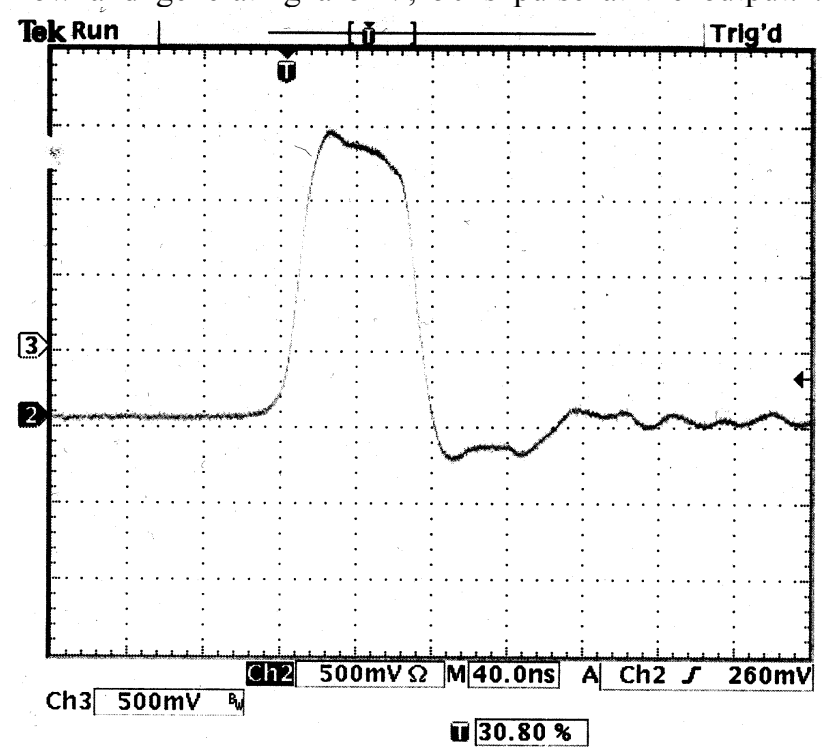

Figure 2: Oscilloscope trace of FID output pulse.

Vertical scale is $5 \mathrm{kV} / \mathrm{div}$; time axis is $40 \mathrm{~ns} / \mathrm{div}$.

The four $5 \mathrm{kV}$ block outputs are combined, using a Blumlein type circuit consisting of four, $6 \mathrm{~m}$ long, $100 \Omega$ coaxial cables, into a single $20 \mathrm{kV}$ positive pulse. The output is matched into a $480 \Omega$ resistive load. The pulse width is fixed at $50 \mathrm{~ns}$ and the pulse height is adjustable from $0-20 \mathrm{kV}$ by varying the $0-300 \mathrm{~V} d c$ input voltage. The pulser output into a $580 \Omega$ resistive load is shown in figure 2. The pulse repetition rate is limited to $50 \mathrm{kHz}$ at the maximum voltage due to component heating.

Since the output consists of positive pulses referenced to ground and the electron gun anode is normally biased at the negative cathode potential $(-13 \mathrm{kV})$, the pulser output must be capacitively coupled to the gun anode through a $3300 \mathrm{pF}, 30 \mathrm{kV}$, ceramic capacitor. The pulser, along with its combining network, $480 \Omega$ resistive load (water and air cooled), and output coupling capacitor is enclosed in a shielded equipment rack to eliminate electrical noise generated in the combining network from interfering with other electronics in the TEL. We have experienced several failures of the HV connectors on the cable between the modulator and the electron gun anode feedthrough due to corona discharges at voltages above $15 \mathrm{kV}$.

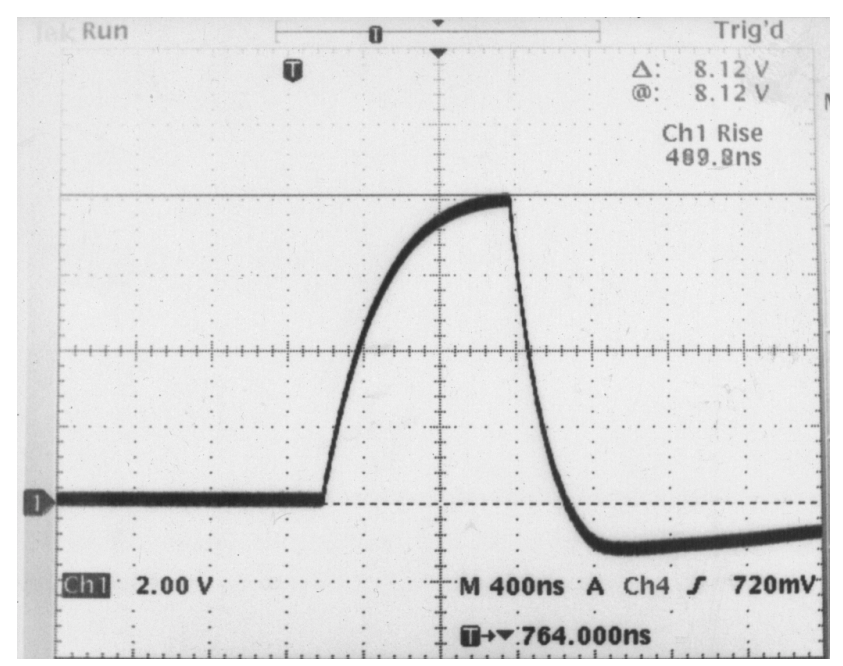

Figure 3: Tetrode modulator output pulse $(2 \mathrm{kV} / \mathrm{div})$ with a sweep time of $400 \mathrm{~ns} / \mathrm{div}$.

\section{TETRODE MODULATOR}

The third type of modulator uses the output from the anode of a grid driven tetrode. The tube anode is connected to a $+10 \mathrm{kV} \mathrm{dc}$ anode supply through a $1500 \Omega$ resistor. The modulating voltage on the anode of the tetrode is then ac-coupled through two $1000 \mathrm{pF}$ ceramic capacitors to the electron gun anode. This modulator has the advantage that it is not susceptible to radiation damage and can be installed directly adjacent to the Tevatron beamline.

A CPI/EIMAC 4cw25000B water-cooled tetrode, with a maximum plate dissipation of $25 \mathrm{~kW}$, is used in this modulator. Its anode voltage is supplied by a Hipotronics $10 \mathrm{kV}, 16 \mathrm{~A}$, dc power supply. An additional LC filter $(1.5 \mathrm{H}, 20 \mu \mathrm{F})$ was added to the output of the Hipotronics supply to reduce ripple to less that 1 part in 10,000 . The anode supply is connected to the tetrode through a $1500 \Omega$, $250 \mathrm{~kW}$, water cooled resistor (Altronic Research). The grid of the tetrode is driven by an Amplifier Research 500A100 amplifier which can provide 500 watts of power from $10 \mathrm{kHz}$ to $100 \mathrm{MHz}$.

For compensating a single bunch of protons or antiprotons, the tube is typically operated with a screen voltage of $750 \mathrm{~V}$ and a dc grid voltage of $-15 \mathrm{~V}$. Under these conditions, the voltage on the anode is held slightly above the screen voltage at $1 \mathrm{kV}$ with a plate current of $6 \mathrm{~A}$. The tetrode's grid is then pulsed with a negative voltage pulse from the broadband amplifier, reducing the current flow through the tetrode. The positive pulse appearing on the anode is then coupled, using two $1000 \mathrm{pF}$ ceramic capacitors in parallel, through a short $(0.6 \mathrm{~m})$ section of 
$50 \Omega, \mathrm{RG} 213$ cable to the anode electrode of the electron gun. A typical output pulse is shown in figure 3. Since the gun anode must be charged through the $1500 \Omega$ resistor, the risetime is limited by the sum of the tetrode's anodescreen capacitance $(35 \mathrm{pF})$, the capacitance of the cable connecting the modulator to the gun $(60 \mathrm{pF})$, and the gun anode to ground capacitance $(60 \mathrm{pF})$. A pulse to pulse amplitude stability of $2 \mathrm{E}-4$ was achieved by applying a feedforward compensation signal to the grid of the tetrode to reduce ripple on the modulator output at power line frequencies. A photograph of the Fermilab built modulator is shown in figure 4 .

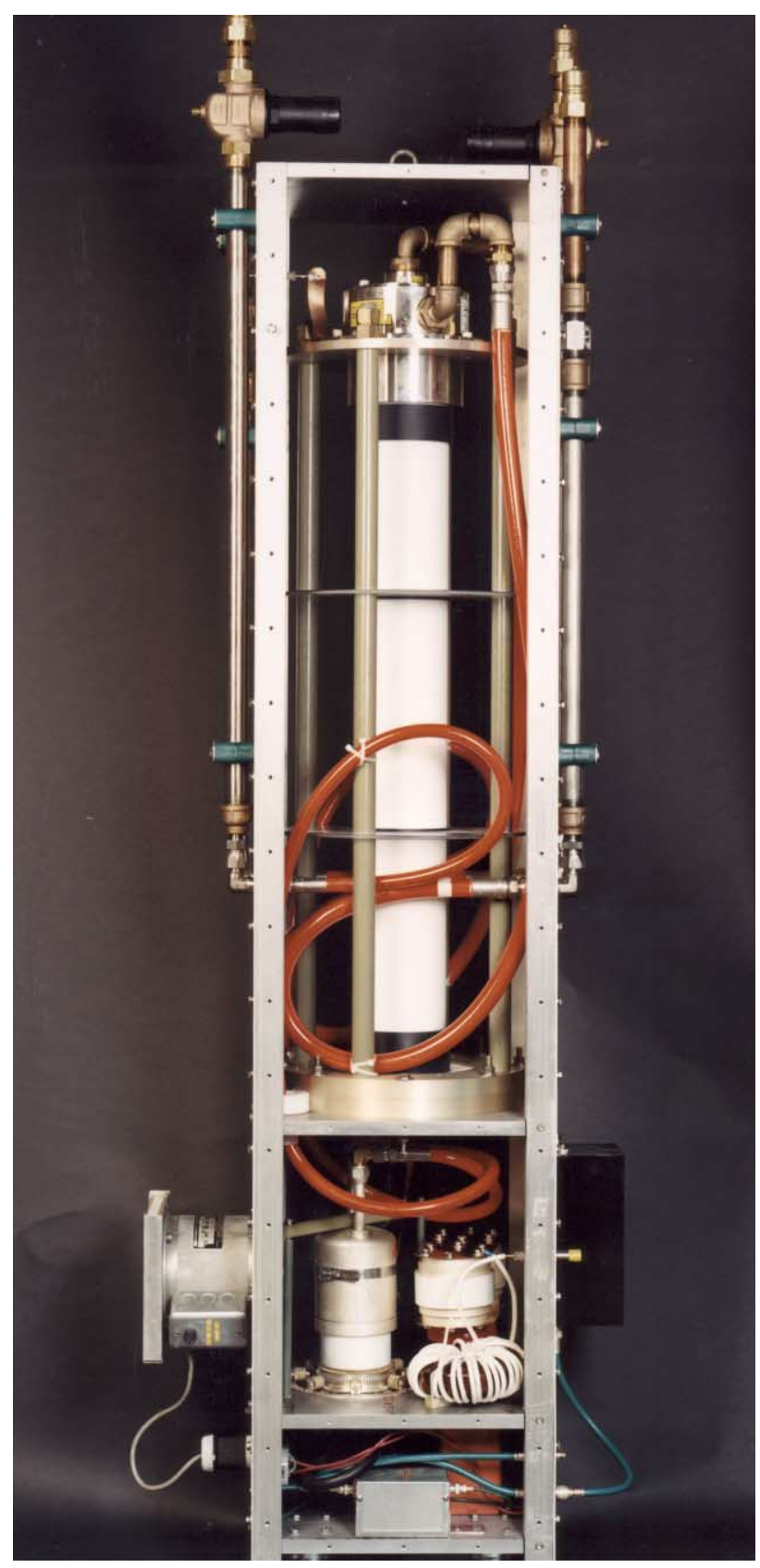

Figure 4: Photograph of the tetrode modulator.

\section{CONCLUSIONS}

Three different modulators have been used for testing the Fermilab Tevatron Electron Lens. Both the MOSFET and Fast Ionization Device modulators produced fast risetime pulses at a $50 \mathrm{kHz}$ repetition rate which could be used for compensating the beam-beam tune shift of a single antiproton bunch in the Tevatron. However; these modulators cannot be used with 36 bunches since their duty factor is limited by component heating.

The tetrode modulator is not duty factor limited so it can compensate multiple bunches. Its output is easily modulated and it can be used in radiation areas. The present disadvantage with this modulator is its relatively slow risetime. This will be improved by $1 / 3$ in the near future by replacing the $1500 \Omega$ load resistor with a $1 \mathrm{k} \Omega$ load. With this modification, the tetrode modulator will meet all of the requirements for the Tevatron Run IIA, 36 X 36 bunch operation, with $395 \mathrm{~ns}$ bunch spacing.

\section{REFERENCES}

[1] V. Shiltsev, et. al., "Beam -Beam Compensation in Tevatron: Status Report," this conference.

[2] N. Solyak, K. Bishofberger, V. Shiltsev, "Electron Beam System of TEL," this conference.

[3] V. Shiltsev, V. Danilov, D. Finley, A. Sery, "Considerations on compensation of beam-beam effects in the Tevatron with electron beams," Phys. Rev. Special Topics - Accel. \& Beams, Vol.2, 1999.

[4] V.M. Efanov, A.V. Kriklenko, P.M. Yarin, N.J. Daviduk, "100kV picosecond all solid state pulser," Proc. 24 ${ }^{\text {th }}$ Intl. Power Modulator Symposium, Norfolk, VA, 2000. 